\title{
Épidémiologie de la tuberculose en milieu urbain, apport de la biologie moléculaire
}

Appliquée à la tuberculose, la biologie moléculaire autorise une nouvelle approche épidémiologique de la maladie [1]. L'analyse du polymorphisme de taille des fragments de restriction du bacille (restriction-fragment-length polymorphism, RFLP) se substitue aux données phénotypiques et permet de différencier des espèces et de "pister" ainsi les circuits épidémiques d'une espèce bacillaire donnée. L'utilisation comme sonde d'un fragment d'ADN particulier, dénommé IS6110, qui est une séquence répétée et spécifique du génome des mycobactéries du complexe $M$. tuberculosis, permet de différencier les souches de $M$. tuberculosis. En effet, l'analyse d'un grand nombre de souches a montré que cette séquence est présente en un nombre de copies variables et à des localisations différentes sur le chromosome. Deux éléments autorisent l'utilisation de cette séquence IS6110 dans un but épidémiologique: d'une part, les transpositions successives de cet élément ont eu pour conséquence de diversifier sa localisation au cours du temps (diversité des souches), d'autre part, l'efficacité de transposition est faible, ce qui entraîne une certaine stabilité du polymorphisme (souche "identique" chez le sujet contaminant et chez le sujet contaminé). La technique utilisée pour détecter le polymorphisme de position de IS6110 consiste donc à cultiver les souches, à extraire l'ADN, à le couper par une enzyme de restriction, puis à détecter les différents fragments contenant une séquence IS6110 à l'aide d'une sonde interne à cette séquence par hybridation des fragments après migration sur gel

génomique (DNA fingerprint) de la souche.

La première application de cette technique a été publiée par Hermans et al. [2] dans la description d'une microépidémie de tuberculose à la suite de l'injection de produits contaminés par $M$. tuberculosis. La possibilité de typer les bacilles a permis de démontrer la transmission d'infections dans divers hôpitaux, dans des lieux d'hébergement ainsi que l'existence de contaminations dans des laboratoires d'analyse $(\mathrm{m} / \mathrm{s}$ $n^{\circ}$ 11, vol. 9, p. 1279).

Cette technique a été utilisée récemment pour décrire l'épidémiologie de la tuberculose dans deux villes des États-Unis : San Francisco [3] et New York [4]. Dans les deux études, un diagnostic RFLP a été réalisé dans un nombre élevé de cas (respectivement 473 et 104); cela est d'un intérêt particulier car déborde du cadre usuel des microépidémies. Dans les deux villes, on note une diversité génétique remarquable parmi les isolats de $M$. tuberculosis, suggérant que la majorité des cas de tuberculose active provient de la réactivation d'une infection ancienne (324 souches distinctes sur 473 à San Francisco [68\%] et 77 sur 104 [74\%] à New York).

Par ailleurs, si on définit une "grappe " comme un ensemble de deux patients, ou plus, qui partagent une même empreinte génomique, on peut supposer, vu la diversité des souches, qu'une grappe n'est pas le fruit d'une coïncidence mais le résultat d'une transmission récente. L'étude épidémiologique classique le confirme dans un grand nombre de cas dans les deux villes. Ainsi, au minimum $26 \%$ à $31 \%$ des patients ont été récemment contaminés. Ces chiffres sont probablement une sousestimation, car certains patients, dont les bacilles présentent une empreinte génomique isolée, peuvent avoir une infection récente mais contractée hors de la zone géographique étudiée. Ces résultats modifient notablement ce qui était généralement admis aux États-Unis où l'on considérait que $10 \%$ seulement des cas de tuberculose étaient dus à une nouvelle infection [5], tous les autres résultant d'une réactivation de foyers infectieux constitués des années, voire des décennies avant l'épisode actuel.

Les facteurs de risque associés à une transmission récente dans les deux villes sont : être jeune, appartenir à une minorité ethnique (hispanique ou noire), être né aux États-Unis, avoir le SIDA. A New York, une transmission récente est également associée à la séropositivité pour le VIH $\left(m / s n^{\circ} 3\right.$, vol. $\left.8, p .288\right)$, à la présence d'une résistance à un ou plusieurs antituberculeux, à un faible revenu.

Près des deux tiers des malades, soit infectés par le VIH, soit sidéens, appartiennent au groupe des patients ayant des souches communes de $M$. tuberculosis; ainsi, chez ces patients, la tuberculose pourrait être plus souvent due à une nouvelle transmission qu'à la réactivation d'une infection lointaine. Cela est également en faveur d'une plus grande sensibilité de ces sujets à l'infection par le BK : une exposition à des bacilles, même ni intense ni prolongée, entraînerait l'infection (et secondairement surviendrait la maladie tuberculeuse). Autre fait remarquable, un seul patient dont le frottis est positif à l'examen direct et reste positif parce que non ou mal 
traité peut être à l'origine d'un très grand nombre de cas secondaires. Le "record " revient à San Francisco, où l'on a estimé qu'un seul sujet présentant ces caractéristiques était responsable de $6 \%$ de tous les cas identifiés dans cette étude.

Au total, l'utilisation de la biologie moléculaire permet d'affiner considérablement notre connaissance de la transmission de la tuberculose : on estime que l'épidémiologie classique n'aurait pu établir un lien entre les cas que pour $10 \%$ d'entre eux. Peut-être cela est-il dû à un changement de l'épidémiologie de la tuberculose en milieu urbain où les chômeurs et les sans-abri sont surreprésentés dans les grappes ainsi que les patients atteints par le VTH. De nouvelles techniques de recherche des sujets contacts et des lieux à risque méritent d'être développées.

L'approche moléculaire - en dehors du fait qu'elle stimule elle-même la recherche épidémiologique et l'intérêt porté à la tuberculose, en général, et aux tuberculeux, en particulier - confirme par ailleurs ce qui est connu de longue date: ce sont les cas à frottis positifs qui sont les principales sources d'infection et la prévention de la survenue d'autres cas passe par une prise en charge correcte de ces cas: dépister est insuffisant, il est impératif de guérir.

A.T.

1. Gicquel B. Épidémiologie moléculaire de la tuberculose. Ann Institut Pasteur 1993 ; 4 : 188-92. 2. Hermans PW'M, Van Soolingen D, Dale JW' Insertion element IS 986 from Mycobacterium tuberculosis : a useful tool for diagnosis and epidemiology of tuberculosis. J Clin Microbiol 1990; 28 : 2051-8.

3. Small PM, Hopewell PC, Singh SP, Paz A, Parsonnet J, Ruston DC, Schecter GF, Daley CI. Schoolnik GK. The epidemiology of tuberculosis in San Francisco. N Engl J Med 1994 ; 330 : 1703-9. 4. Alland D, Kalkut GE, Moss AR, McAdam RA. Hahn JA, Bosworth W', Drucker E, Bloom BR. Transmission of tuberculosis in New York city. $N$ Engl J Med 1994 ; 330 : 1710-6.

5. Horwize (), Edwards PQ, L.owell AM. National tuberculosis control program in Denmark and the United States. Health Sern' Rep) 1973 ; 88 : 493-8.

\section{BRÈVES}

$\square$ GATA-4, régulateur potentiel de la différenciation cardiaque. L'étude des facteurs de transcription myogéniques appartenant à la famille MyoD a non seulement permis une meilleure compréhension du contrôle de l'expression des gènes musculaires mais a contribué de façon remarquable à élaborer les mécanismes de la différenciation du muscle squelettique. Cependant, le contrôle de la transcription et les mécanismes de différenciation d'un autre muscle strié, le muscle cardiaque, semblent être distincts puisque les facteurs myogéniques qui sont cruciaux pour le développement du muscle squelettique ne sont pas présents dans le cœur, et l'inactivation de leurs gènes par recombinaison homologue n'a aucun effet sur le développement cardiaque. Puisque l'expression des gènes spécifiques d'un tissu est une composante importante de la différenciation cellulaire, l'étude de la transcription des gènes spécifiques du tissu cardiaque devrait permettre d'identifier les facteurs de transcription spécifiques du cœur qui pourraient être également des facteurs de différenciation. C'est l'approche adoptée par l'équipe de Mona Nemer (IRCM, Montréal, Canada) qui, en étudiant la régulation transcriptionnelle du gène cardiaque $b n p$ (peptide natriurétique de type $\mathrm{B})$, a isolé un facteur de transcription cardiaque appartenant à la famille des facteurs GATA dont les trois membres déjà connus sont spécifiques des cellules hématopoïétiques [1]. Les auteurs ont montré que l'activité cardiaque spécifique du promoteur bnp était relayée par des motifs GATA situés dans les 114 premières pb de séquences régulatrices en 5 '. Le criblage d'une banque d'ADN complémentaire issue de cardiomyocytes a permis d'isoler un ADNc codant pour une protéine (GATA4) qui contient un motif en doigt de zinc et qui est $70 \%$ similaire aux autres membres de la famille GATA. Le gène (sATA-4 est trans- crit abondamment dans les cardiomyocytes et les gonades uniquement. Par ailleurs, les gènes des peptides natriurétiques de type A (ANP) ou B (BNP) qui sont exprimés dans les myocytes cardiaques mais non squelettiques sont des cibles de la protéine GATA-4 [1]. Ainsi, GATA-4 serait le premier facteur de transcription cardiaque dont les gènes cibles sont clairement identifiés. Plus récemment, l'équipe de Bruce Markham (Medical College of Wisconsin, USA) [2] a démontré que deux motifs GATA dans le promoteur du gène cardiaque MHC (myosin heavy chain, chaîne lourde de la myosine) sont fonctionnellement importants pour l'activité cardiaque du promoteur dans les cardiomyocytes. La protéine qui relaie cette activité transcriptionnelle a été identifiée par critères immunologiques comme étant GATA-4. Ainsi, trois gènes cardiaques codant pour des peptides hormonaux et pour une protéine structurale sont activés par GATA-4. Des motifs GATA sont également présents dans les promoteurs d'autres gènes cardiaques incluant l'actine cardiaque, les isoformes cardiaques des chaînes légères des myosines (MLC-1/A et MLC-1/V) ainsi que la troponine cardiaque de type C, suggérant que GATA-4 pourrait être un activateur global du programme génétique cardiaque. Ainsi, le facteur de transcription GATA-4 est un activateur transcriptionnel cardiaque à distribution tissulaire très restreinte. Ce comportement, spécificité tissulaire et rôle d'activateur transcriptionnel, est analogue aux comportements des facteurs myogéniques de la famille héliceboucle-hélice et du facteur GATA1, impliqué dans la différenciation des cellules érythroïdes, laissant clairement entrevoir un rôle de GATA-4 dans la différenciation du muscle cardiaque.

[1. Grépin C, et al. Mol Cell Biol 1994 ; 14 : 3115-29.]

[2. Molkentin JD, et al. Mol Cell Biol 1994 ; 14 : 4947-57.]
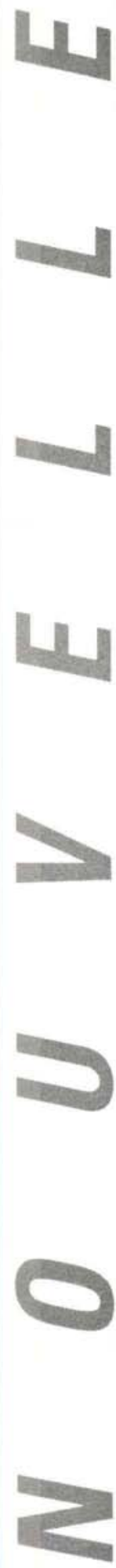